\title{
Analysis on a General Class of Holographic Type Dark Energy Models
}

\author{
Zhuo-Peng Huang ${ }^{1,2, *}$ and Yue-Liang $\mathrm{Wu}^{2,+}$ \\ ${ }^{1}$ Department of Physics, National University of Defense Technology, Hunan 410073, China \\ ${ }^{2}$ State Key Laboratory of Theoretical Physics (SKLTP) \\ Kavli Institute for Theoretical Physics China (KITPC) \\ Institute of Theoretical Physics, Chinese Academy of Sciences, Beijing 100190, China
}

(Dated: June 13, 2018)

We present a detail analysis on a general class of holographic type dark energy models characterized by the length scale $L=\frac{1}{a^{n}(t)} \int_{0}^{t} d t^{\prime} a^{m}\left(t^{\prime}\right)$. We show that $n \geq 0$ is required by the recent cosmic accelerated expansion of universe. In the early universe dominated by the constituent with constant equation of state $w_{m}$, we have $w_{d e} \simeq-1-\frac{2 n}{3}$ for $n \geq 0$ and $m<0$, and $w_{d e} \simeq-\frac{2}{3}(n-m)+w_{m}$ for $n>m \geq 0$. The models with $n>m \geq 0$ become single-parameter models like the $\Lambda \mathrm{CDM}$ model due to the analytic feature $\Omega_{d e} \simeq$ $\frac{d^{2}}{4}\left(2 m+3 w_{m}+3\right)^{2} a^{2(n-m)}$ at radiation- and matter-dominated epoch. Whereas the cases $n=m \geq 0$ should be abandoned as the dark energy cannot dominate the universe forever and there might be too large fraction of dark energy in early universe, and the cases $m>n \geq 0$ are forbidden by the self-consistent requirement $\Omega_{d e} \ll 1$ in the early universe. Thus a detailed study on the single-parameter models corresponding to cases $n>m \geq 0$ is carried out by using recent observations. The best-fit analysis indicates that the conformal-age-like models with $n=m+1$, i.e. $L \propto \frac{1}{H a}$ in early universe, are more favored and also the models with smaller $n$ for the given $n-m$ are found to fit the observations better. The equation of state of the dark energy in models with $n=m+1>0$ transits from $w_{d e}<-1$ during inflation to $w_{d e}>-1$ in radiation- and matter-dominated epoch, and then back to $w_{d e}<-1$ eventually. The best-fit result of the case $(n=0, m=-1)$ which is so-called $\eta$ HDE model proposed in [1] is the most favorable model and compatible with the $\Lambda$ CDM model.

PACS numbers:

*Electronic address: zphuang@ nudt.edu.cn

†Electronic address: ylwu@itp.ac.cn 


\section{INTRODUCTION}

Since the discovery of the current accelerated expansion of the universe [2, 3], many efforts have been made to explain the physical essence behind this phenomenon of acceleration [4-9]. Within the framework of the general relativity, the acceleration can be attributed to the existence of a mysterious negative pressure component named phenomenally as dark energy. A positive cosmological constant, which fits to the observations well, is currently still the simplest candidate for dark energy. However, it is plagued with the fine-tuning problem and the coincidence problem [10]. According to the holographic principle [11, 12] that the number of degrees of freedom of a physical system scales with the area of its boundary rather than its volume. This might indicate that degrees of freedom are overcounted in the calculation of the vacuum energy in effective field theory. The author in [13] suggested that the ultraviolet (UV) cutoff $\Lambda_{\mathrm{uv}}$ in the effective field theory should be related to the infrared (IR) cutoff $L$ due to the limit set by the formation of a black hole, i.e., in terms of the natural units,

$$
L^{3} \Lambda_{\mathrm{uv}}^{4} \lesssim L M_{p}^{2}
$$

where $M_{p}^{2}=1 /(8 \pi G)$ with $G$ the Newton's constant. Such relation leads to a dramatic depletion of quantum states, which results in a much small vacuum energy density,

$$
\rho_{\mathrm{vac}} \sim \Lambda_{\mathrm{uv}}^{4} \sim M_{p}^{2} L^{-2}
$$

When the IR cutoff $L$ is comparable to the current Hubble radius, the fine-tuning problem of the cosmological constant would not exist [13]. Some interesting studies on cosmology with holography have been carried out [14-17]. In ref.[1], we have shown that when the IR cutoff $L$ is characterized by the total comoving horizon of the universe, the inflation [18-21] in early universe makes the vacuum energy given in Eq.(2) behave as a cosmological constant without the fine-tuning problem as well as the coincidence problem, which provided an interesting insight on the cosmological constant as the simplest and best candidate for the dark energy. In alternative, we have found in [22, 23] that when the IR cutoff $L$ is taken to be a conformal-age-like length, the dark energy density given by the vacuum energy in Eq.(2) is almost insensitive to the inflation in early universe.

In the spirit of the holographic principle [11-13], some interesting holographic dark energy models in which the dark energy density is assumed to scale as $\rho_{d e} \propto M_{P}^{2} L^{-2}$ with $L$ being some characteristic length scale of the universe have been proposed and studied by many groups [24-48]. 
Particularly, the first holographic dark energy model (HDE) was proposed in [25] by identifying $L$ with the radius of the future event horizon, i.e. $a(t) \int_{t}^{\infty} \frac{d t^{\prime}}{a\left(t^{\prime}\right)}$, here $a(t)$ is the scale factor of the universe at cosmic time $t$. Alternatively, the total comoving horizon $\int_{0}^{t} \frac{d t^{\prime}}{a\left(t^{\prime}\right)}$ including the very large primordial part generated by inflation was taken in [1] as the IR cutoff to establish the so-called $\eta \mathrm{HDE}$ model, as a consequence, the holographic dark energy behaves almost like a cosmological constant. On the other hand, a conformal-age-like length $\frac{1}{a^{4}(t)} \int_{0}^{t} d t^{\prime} a^{3}\left(t^{\prime}\right)$ motivated from the four dimensional spacetime volume at cosmic time $t$ of the flat FRW universe was adopted in [22] to build the holographic type dark energy model(CHDE). Based on different physical origin, the age $\int_{0}^{t} d t^{\prime}$ of the universe is chosen as the IR cutoff to construct the agegraphic dark energy model(ADE) in [36], its improved new version(NADE) was proposed with replacing the age of the universe by the conformal age $\int^{t} \frac{d t^{\prime}}{a\left(t^{\prime}\right)}$ of the universe [37].

From the above considerations, it is interesting to investigate a general class of holographic type dark energy models with the characteristic length scale given by the following form

$$
L=\frac{1}{a^{n}(t)} \int_{0}^{t} d t^{\prime} a^{m}\left(t^{\prime}\right)
$$

Here we will focus on the case with $n, m$ being the integers. Obviously, the ADE model [36] corresponds to the case $(n=0, m=0)$; the $\eta \mathrm{HDE}$ model [1] corresponds to $(n=0, m=-1)$, while the NADE model [37] corresponds to the same case but without considering a primordial part generated by inflation[1]; the CHDE model [22] corresponds to $(n=4, m=3)$.

In this note, we are going to investigate in detail a general class of holographic type dark energy models characterized by the IR cutoff given in Eq.(3). Our main purpose is to see the possible holographic type dark energy candidates for various cases of $(n, m)$ by requiring the corresponding models to be self-consistent and also consistent with the expansion history of the universe. In particular, we will show which cases are more favorable. In Sec. II, we first present a description on the general class of holographic type dark energy models, and then we make a detail investigation on various models and find out the possible candidates for the various choices $(n, m)$; In Sec. III, we will mainly focus on the single-parameter models similar to the $\Lambda \mathrm{CDM}$ model and perform the best-fit analysis by using recent cosmological observations; Some concluding remarks and discussions are given in Sec. IV. 


\section{A GENERAL CLASS OF HOLOGRAPHIC TYPE DARK ENERGY MODELS}

Let us begin with the general characteristic length scale of the universe

$$
L=\frac{1}{a^{n}(t)} \int_{0}^{t} d t^{\prime} a^{m}\left(t^{\prime}\right)=\frac{1}{a^{n}(t)} \int_{0}^{a} a^{\prime m} \frac{d a^{\prime}}{H^{\prime} a^{\prime}},
$$

where $H \equiv \dot{a} / a$ is the Hubble parameter and $\cdot$ denotes the derivative with respect to the cosmic time $t$. The corresponding holographic type dark energy density is parameterized by

$$
\rho_{d e}=3 d^{2} M_{p}^{2} L^{-2}
$$

where $d$ is a positive constant parameter. The fractional energy density is defined by

$$
\Omega_{d e}=\frac{\rho_{d e}}{3 M_{p}^{2} H^{2}}=\frac{d^{2}}{H^{2} L^{2}} .
$$

For simplicity, let us consider a flat Friedmann-Robertson-Walker (FRW) universe containing the holographic type dark energy and ambient constituent with constant equation of state (EoS) $w_{m}$. The Friedmann equation is given by

$$
3 M_{p}^{2} H^{2}=\rho_{m}+\rho_{d e},
$$

or in fractional energy densities

$$
\Omega_{d e}+\Omega_{m}=1,
$$

where $\Omega_{m}=\frac{\rho_{m}}{3 M_{p}^{2} H^{2}}$. If there is no direct energy interchange, each energy component is conservative respectively, which results in conservation equations given by

$$
\dot{\rho}_{i}+3 H\left(1+w_{i}\right) \rho_{i}=0
$$

with $i=m, d e$. By using Eqs.(4), (5), (6) and (9), the EoS of the holographic type dark energy is found to be

$$
w_{d e}=-1-\frac{2}{3} n+\frac{2}{3 d} \sqrt{\Omega_{d e}} a^{m-n} .
$$

Cosmic acceleration requires that $w_{d e}<-\frac{1}{3}$ recently, which indicates $n>-1$ or equivalently $n \geq 0$ as $n$ is taken to be integer under our present consideration.

The conservation of the ambient constituent with constant $w_{m}$ leads to $\rho_{m}=C_{1} a^{-3\left(1+w_{m}\right)}$, where $C_{1}$ is a constant coefficient. Combining with the Friedmann equation and the definition of fractional energy densities, we have

$$
\frac{1}{H a}=\frac{1}{\sqrt{\frac{c_{1}}{3 M_{p}^{2}}}} \sqrt{a^{\left(1+3 w_{m}\right)}\left(1-\Omega_{d e}\right)} .
$$


By using Eqs.(4) and (6), we get

$$
\int_{0}^{a} a^{\prime m} \frac{d a^{\prime}}{H^{\prime} a^{\prime}}=\frac{a^{n+1} d}{\sqrt{\Omega_{d e}} H a} .
$$

Substituting Eq.(11) into above equation and taking derivative with respect to $a$ in both sides, we arrive at the differential equation of motion for $\Omega_{d e}$

$$
\frac{d \Omega_{d e}}{d a}=\frac{\Omega_{d e}}{a}\left(1-\Omega_{d e}\right)\left(3\left(1+w_{m}\right)+2 n-\frac{2}{d} \sqrt{\Omega_{d e}} a^{m-n}\right) .
$$

Obviously, under the transformation $a \rightarrow \frac{a}{a_{0}}, d \rightarrow d a_{0}^{n-m}$, with taking $a_{0}$ as the present scale factor of the universe, the energy density Eq.(5), the fractional energy density Eq.(6), the EoS Eq.(10) and the differential equation Eq.(13) are all invariant. Namely, performing such transformation, all expressions keep the same, so we can set $a_{0}=1$. From now on, we adopt that the parameter $d$ has absorbed a factor $a_{0}^{n-m}$ and set $a_{0}=1$. Note that the subscript " 0 " always indicates the present value of the corresponding quantity.

Considering that our universe has successively experienced the inflation during which $w_{m} \simeq-1$ (which indicates quasi-de Sitter expansion), the radiation-dominated epoch during which $w_{m}=\frac{1}{3}$, and the matter-dominated epoch during which $w_{m}=0$ before turning to accelerated expansion recently, we are actually able to approximately study the behaviors of $L$, thus the fractional density $\Omega_{d e}$ in the early universe directly. Here, we will simply ignore the effect due to the transition from one ambient-constituent-dominated epoch to another ambient-constituent-dominated epoch.

Let us define

$$
\tilde{L}=\frac{1}{a^{m}(t)} \int_{0}^{t} d t^{\prime} a^{m}\left(t^{\prime}\right)=\left(\frac{a_{i}}{a}\right)^{m} \tilde{L}_{i}+\frac{1}{a^{m}(t)} \int_{t_{i}}^{t} d t^{\prime} a^{m}\left(t^{\prime}\right), \quad \tilde{L}_{i}=\frac{1}{a_{i}^{m}} \int_{0}^{t_{i}} d t^{\prime} a^{m}\left(t^{\prime}\right)
$$

where the subscript $i$ denotes the beginning of some epoch under consideration. Obviously, we have $L=a^{m-n} \tilde{L}$.

When the constituent with constant $w_{m}$ dominates the universe from $t_{i}$, we have approximately $H^{2} \propto \rho_{m} \propto a^{-3\left(1+w_{m}\right)}$ from Fridemann equations, which results in the following consequences

$$
\begin{aligned}
& \frac{1}{a^{m}(t)} \int_{t_{i}}^{t} d t^{\prime} a^{m}\left(t^{\prime}\right)=\frac{1}{a^{m}(t)} \int_{a_{i}}^{a} a^{\prime m} \frac{d a^{\prime}}{H^{\prime} a^{\prime}} \\
&=\left\{\begin{array}{cl}
\frac{1}{m+\frac{3\left(1+w_{m}\right)}{2}}\left(\frac{1}{H}-\frac{1}{H_{i}}\left(\frac{a_{i}}{a}\right)^{m}\right) & m>-\frac{3\left(1+w_{m}\right)}{2}, \\
\frac{1}{H} \ln \left(\frac{a}{a_{i}}\right) & m=-\frac{3\left(1+w_{m}\right)}{2}, \\
\frac{1}{\left|m+\frac{3\left(1+w_{m}\right)}{2}\right|}\left(\frac{1}{H_{i}}\left(\frac{a_{i}}{a}\right)^{m}-\frac{1}{H}\right) & m<-\frac{3\left(1+w_{m}\right)}{2} .
\end{array}\right.
\end{aligned}
$$




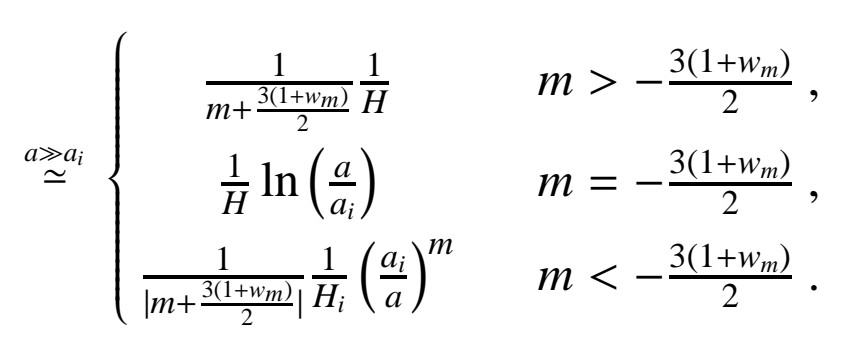

Noticing that the approximation in the limit $a \gg a_{i}$ is due to the fact that $H^{2} \propto a^{-3\left(1+w_{m}\right)}$ and

$$
\frac{1}{H_{i}}\left(\frac{a_{i}}{a}\right)^{m} / \frac{1}{H}=\left(\frac{a_{i}}{a}\right)^{m+\frac{3\left(1+w_{m}\right)}{2}}
$$

When $m>0$, then $m>-\frac{3\left(1+w_{m}\right)}{2}$ all the time in the early universe. From Eqs.(14) and (15), we have

$$
\tilde{L}=\frac{1}{a^{m}(t)} \int_{0}^{t} d t^{\prime} a^{m}\left(t^{\prime}\right)=\left(\frac{a_{i}}{a}\right)^{m} \tilde{L}_{i}+\frac{1}{m+\frac{3\left(1+w_{m}\right)}{2}}\left(\frac{1}{H}-\frac{1}{H_{i}}\left(\frac{a_{i}}{a}\right)^{m}\right)
$$

for the constituent with constant $w_{m}$ dominating the universe. During the inflation, the Hubble parameter $H$ is constant approximately and the universe expands exponentially. Those terms with the factor $\left(\frac{a_{i}}{a}\right)^{m}$ will soon become negligible, we have $\tilde{L} \simeq \frac{1}{m+\frac{3\left(1+w_{m}\right)}{2}} \frac{1}{H}$ approximately, which also results in $\tilde{L}_{e} \sim O\left(1 / H_{e}\right)$ at the end of the inflation. Approximately, for the radiation dominated epoch, the initial value $\tilde{L}_{i} \sim \tilde{L}_{e}$ is at the order of $O\left(1 / H_{e}\right)$. Referring to Eq.(16) for $m>-\frac{3\left(1+w_{m}\right)}{2}$ and reminding that the expansion of the universe, we have $\tilde{L} \simeq \frac{1}{m+\frac{3\left(1+w_{m}\right)}{2}} \frac{1}{H}$ for radiation-dominated epoch during which $w_{m}=\frac{1}{3}$ as well. Similar result holds during the matter-dominated era. By using $L=a^{m-n} \tilde{L}$ and Eq.(6), we get the approximate fraction of dark energy in early universe, i.e.

$$
\Omega_{d e} \simeq \frac{d^{2}}{4}\left(2 m+3 w_{m}+3\right)^{2} a^{2(n-m)},
$$

where $w_{m} \simeq-1$ during inflation, $w_{m}=\frac{1}{3}$ in radiation-dominated epoch and $w_{m}=0$ in matterdominated epoch respectively. For self-consistency, we need $n>m$ to ensure that $\Omega_{d e} \ll 1$ when $a \ll 1$, thus the ambient matter dominated the early universe. It is not difficult to prove that for $n>m$, Eq.(18) is the approximate solution of the differential equation of $\Omega_{d e}$ under the limit $1-\Omega_{d e} \simeq 1$ when $a \ll 1$ consistently.

For the case $m=0$, from Eqs.(14) and (15), we simply have $\Omega_{d e} \simeq d^{2} a^{2 n}\left(\ln \left(\frac{a}{a_{i}}\right)\right)^{-2}$ during inflation. While the approximate solution in matter- or radiation-dominated epoch is also given by Eq.(18). Thus $n>m$ is also required to be self-consistent.

Let us now pay attention to the case $n=m \geq 0$. In this case, we have $\Omega_{d e} \equiv \frac{d^{2}}{4}\left(2 m+3 w_{m}+3\right)^{2}$ at the matter-dominated epoch, which is the exact solution of the differential equation. Referring to the EoS of dark energy Eq.(10), we get $w_{d e} \equiv w_{m}$ which means that the holographic type dark 
energy tracks the dominated component and never dominates. This is of course unacceptable. Moreover, unless the parameter $d$ is small enough, the faction of dark energy would be too large in early universe to be consistent with primordial nucleosynthesis (BBN) [49]. While by referring to the EoS Eq.(10), a tiny $d$ would make the present EoS of dark energy $w_{d e 0}$ severely deviating from -1 and be inconsistent with recent observations [50].

For the case $m<-2$, then $m<-\frac{3\left(1+w_{m}\right)}{2}$ holds all the time in the early universe. From Eqs.(14), (15) and Eq.(16), we have in early universe

$$
\tilde{L} \simeq\left(\tilde{L}_{i}+\frac{1}{\left|m+\frac{3\left(1+w_{m}\right)}{2}\right|} \frac{1}{H_{i}}\right)\left(\frac{a_{i}}{a}\right)^{m} \gg \frac{1}{H} .
$$

Combining with $L=a^{m-n} \tilde{L}$ and $H^{2} \propto \rho_{m} \propto a^{-3\left(1+w_{m}\right)}$, we get the fraction of dark energy in early universe when $a_{i} \ll a \ll 1$

$$
\Omega_{d e}=\frac{d^{2}}{H^{2} L^{2}}=\left(H^{-1} / \tilde{L}\right)^{2} d^{2} a^{2(n-m)} \propto d^{2}\left(a_{i}\right)^{2|m|} a^{3\left(1+w_{m}\right)+2 n} .
$$

As it is analyzed above that $n \geq 0$ is required by recent cosmic acceleration, we then have $\Omega_{d e} \ll 1$ when $a \ll 1$ consistently for $m<0$ here. The scaling property of $\Omega_{d e}$ with respect to $a$ can also be resulted from the differential equation of $\Omega_{d e}$ under the limit $1-\Omega_{d e} \simeq 1$ when $a \ll 1$. This is because in light of the above equation the last factor in the differential equation Eq.(13) is found to be

$$
\frac{2}{d} \sqrt{\Omega_{d e}} a^{m-n}=\frac{2}{H L} a^{m-n}=2 H^{-1} / \tilde{L} \ll 1,
$$

which is negligible small.

For the cases $m=-1$ and $m=-2$, we can also obtain consistently Eq.(20) for $n \geq 0$ during the inflation by following the same argument for the case $m<-2$. While for the radiation- and matter-dominated era, more attention is needed. Let us rewrite $\tilde{L}$ in the following way,

$$
\tilde{L}=\frac{1}{a^{m}(t)} \int_{t_{b}}^{t_{e}} d t^{\prime} a^{m}\left(t^{\prime}\right)+\frac{1}{a^{m}(t)} \int_{t_{e}}^{t} d t^{\prime} a^{m}\left(t^{\prime}\right),
$$

where subscripts $b$ and $e$ denote the beginning and the end of inflation respectively. From Eq.(15), it is not difficult to find that the second term $\frac{1}{a^{m}(t)} \int_{t_{e}}^{t} d t^{\prime} a^{m}\left(t^{\prime}\right)$ is approximately at order of $O(1 / H)$ for the cases $m=-1$ and $m=-2$, while the first term is given by

$$
\frac{1}{a^{m}(t)} \int_{t_{b}}^{t_{e}} d t^{\prime} a^{m}\left(t^{\prime}\right) \simeq \frac{1}{|m|} \frac{a}{H_{b} a_{b}}\left(\frac{a}{a_{b}}\right)^{|m|-1} .
$$

In order to solve the horizon problem [18], the inflation is required to last enough time to make

$$
\frac{1}{H_{b} a_{b}}>\frac{1}{H_{0} a_{0}} \text {. }
$$


Moreover, due to the fact that $\frac{1}{H a}$ has been growing as $a^{(1+3 w) / 2}$ until recent cosmic acceleration (from then on, $w<-\frac{1}{3}$ ), we generally have $\frac{1}{H_{0} a_{0}} \gg \frac{1}{H a}$ when $a_{e} \ll a \ll a_{0}$. Thus for $m \leq-1$, we yield

$$
\frac{1}{|m|} \frac{1}{H_{b} a_{b}}\left(\frac{a}{a_{b}}\right)^{|m|-1}>\frac{1}{H_{0} a_{0}} \gg \frac{1}{H a},
$$

which indicates that for the cases $m=-1$ and $m=-2$, the first term in Eq.(22) is much larger than the second term. Thus we approximately have

$$
\tilde{L} \simeq \frac{1}{a^{m}(t)} \int_{t_{b}}^{t_{e}} d t^{\prime} a^{m}\left(t^{\prime}\right) \gg \frac{1}{H}
$$

in the radiation- and matter-dominated epoch. Therefore, Eq.(20) also holds at these two epoches for $n \geq 0$ and $m=-1$ or $m=-2$.

It is noticed that for the case $n=0$ and $m<0$ the characteristic scale $L$ will be dominated by the primordial part generated in inflation with referring to Eqs.(4), (15), (25), i.e.

$$
L=\int_{0}^{t} d t^{\prime} a^{m}\left(t^{\prime}\right) \simeq L_{\text {prim }}=\int_{t_{b}}^{t_{e}} d t^{\prime} a^{m}\left(t^{\prime}\right) \simeq \frac{1}{|m|} \frac{1}{H_{b} a_{b}}\left(\frac{1}{a_{b}}\right)^{|m|-1}
$$

in the radiation- and matter-dominated epoch. Then the dark energy behaves almost like a cosmological constant during these two epoches with

$$
\rho_{d e} \simeq 3 d^{2} M_{p}^{2} L_{\text {prim }}^{-2}
$$

Correspondingly, the fractional energy density of dark energy scales as

$$
\Omega_{d e} \simeq \frac{d^{2}}{H^{2} L_{\text {prim }}^{2}} \propto \frac{d^{2}}{L_{\text {prim }}^{2}} a^{3\left(1+w_{m}\right)} .
$$

The $\eta \mathrm{HDE}$ model corresponding to the case $(n=0, m=-1)$ has been investigated in [1]. In the cases with $m<0$, the observation constraints on the parameter $d$ is very weak and the models seemly have only one effective parameter, i.e. the ratio $L / d \sim O\left(H_{0}^{-1}\right)$. In the $\eta \mathrm{HDE}$ model, the parameter $d$ can take value in a normal order [1]. While for the case $m<-1$, the parameter $d$ seems to take very large value due to the very large factor in $L$, i.e. $\left(\frac{1}{a_{b}}\right)^{|m|-1}$, in order to make the ratio $L / d$ in the right order.

In summary, $n \geq 0$ is required by the recent cosmic accelerated expansion. For $n \geq 0$ and $m<0$, the fraction of dark energy density scales as $\Omega_{d e} \propto a^{3\left(1+w_{m}\right)+2 n}$ with a tiny proportionality coefficient in the early universe when $a \ll 1$. Since the proportionality coefficient can not be determined only by the parameter $d$, there are in general two model parameters. They may be 
chosen to be the parameter $d$ and the present fraction of dark energy $\Omega_{d e 0}$. However, if $n=0$, the holographic type dark energy would behave almost like a cosmological constant and there seems to be only one effective parameter $\Omega_{d e 0}$. Since the total energy density $\rho=3 M_{p}^{2} H^{2} \propto a^{-3\left(1+w_{m}\right)}$ approximately when the constituent with constant $w_{m}$ dominates the universe, we have $\rho_{d e} \propto a^{2 n}$. Thus $w_{d e} \simeq-1-\frac{2 n}{3}$ in the early universe.

For $n>m>0$, we have $\Omega_{d e} \simeq \frac{d^{2}}{4}\left(2 m+3 w_{m}+3\right)^{2} a^{2(n-m)}$ in the early universe when $a \ll 1$. For $n>m=0$, we also have $\Omega_{d e} \simeq \frac{d^{2}}{4}\left(2 m+3 w_{m}+3\right)^{2} a^{2(n-m)}$ in the matter- or radiation-dominated epoch, while $\Omega_{d e} \simeq d^{2} a^{2 n}\left(\ln \left(\frac{a}{a_{i}}\right)\right)^{-2}$ during inflation.

It is seen that for all cases $n>m \geq 0$ the fraction of dark energy can be ignored naturally in early universe when $a \ll 1$ as long as the parameter $d$ takes a normal order value. Moreover, due to such analytic feature, we can use the approximate solution at some $a_{\mathrm{ini}} \ll 1$ in matterdominated epoch as the initial condition to solve the equation of motion for $\Omega_{d e}$. Noticing that once $d$ is given, the present fractional energy density $\Omega_{d e}(a=1)$ can be obtained. So the model is a single-parameter model like the $\Lambda \mathrm{CDM}$ model. Substituting this approximate solution to the EoS of dark energy Eq.(10), we get

$$
w_{d e}=-\frac{2}{3}(n-m)+w_{m}
$$

in the ambient-constituent-dominated epoch. Referring to Eq.(10), $w_{d e}$ will transit to $-1-\frac{2 n}{3}$ due to the expansion of the universe. Obviously, for models with $n=m+1>0$, the EoS of the dark energy in the inflation, radiation- and matter-dominated epoch are $-\frac{5}{3},-\frac{1}{3}$ and $-\frac{2}{3}$ respectively, and transit to $-1-\frac{2 n}{3}$ eventually. Therefore, the EoS of dark energy in such kinds of models transit from $w_{d e}<-1$ to $w_{d e}>-1$ and back to $w_{d e}<-1$ during the universe expansion.

The choices $n=m \geq 0$ are abandoned because the dark energy cannot dominate the universe forever and there might be too large fraction of dark energy in early universe. While the choices $m>n \geq 0$ are forbidden by the self-consistent requirement that $\Omega_{d e} \ll 1$ when $a \ll 1$.

We are going to mainly focus on the single-parameter models corresponding to the cases $n>$ $m \geq 0$. In next section, we will perform the best-fit analyses on such kinds of models by using recent observations. As the $\eta \mathrm{HDE}$ model [1] corresponding to the case $(n=0, m=-1)$ has only one effective parameter, we shall also take it into consideration for a comparison. 


\section{OBSERVATIONAL CONSTRAINTS ON SINGLE-PARAMETER MODELS}

In this section, we are going to mainly focus on the single-parameter models corresponding to the cases $n>m \geq 0$ and perform best-fit analyses on them by using recent cosmological observations including the Union2.1 compilation of 580 supernova Ia (SNIa) data [50], the parameter $A$ from BAO measurements[51] and the shift parameter $R$ from CMB measurements [52]. The observational data and analysis method are given in Appendix A.

As the cosmological observations mainly come from the epoch when the fraction of radiation energy is tiny, we then simply consider a flat Friedmann-Robertson-Walker (FRW) universe containing only dark energy and matter with $w_{m}=0$. Using $a=\frac{1}{1+z}$ with $z$ the redshift, we can rewrite Eq.(13) as

$$
\frac{d \Omega_{d e}}{d z}=-\frac{\Omega_{d e}\left(1-\Omega_{d e}\right)}{1+z}\left(2 n+3-\frac{2}{d} \sqrt{\Omega_{d e}}(1+z)^{n-m}\right)
$$

and the approximate solution for the cases $n>m \geq 0$ in the matter-dominated epoch is given by

$$
\Omega_{d e} \simeq \frac{d^{2}}{4}(2 m+3)^{2}(1+z)^{-2(n-m)},
$$

which can be chosen as an approximate solution at some $z_{\text {ini }}$. Namely, we may take $\Omega_{d e}\left(z_{i n i}\right)=$ $\frac{d^{2}}{4}(2 m+3)^{2}\left(1+z_{\text {ini }}\right)^{-2(n-m)}$ as the initial condition to solve the differential equation Eq. (31). The solution depends weakly on the choice of $z_{i n i}$ in a wide range, since $\Omega_{d e}$ is tiny and scales as $(1+z)^{-2(n-m)}$ when $z \gg 1$. Here, we simply take $z_{\text {ini }}=2000$ at which matter dominates the universe well.

As the $\eta \mathrm{HDE}$ model [1] corresponding to the case $(n=0, m=-1)$ behaves as a single effective parameter model, we would like to take it into consideration for a comparison. Reminding that there are actually two parameters in this model, we may choose $d$ and $\Omega_{m}(z=0)$ as two parameters. Taking $\Omega_{d e}(z=0)=1-\Omega_{m}(z=0)$ as the boundary condition, we are able to solve the differential equation Eq.(31).

From the Friedmann equation and conservative equations, we have

$$
H^{2}(z)=H_{0}^{2} \Omega_{m 0}(1+z)^{3}+H^{2}(z) \Omega_{d e}(z) .
$$

Equivalently,

$$
E(z) \equiv \frac{H(z)}{H_{0}}=\left(\frac{\Omega_{m 0}(1+z)^{3}}{1-\Omega_{d e}(z)}\right)^{1 / 2} .
$$

Substituting the results of $\Omega_{m 0}=1-\Omega_{d e}(z=0)$ and $\Omega_{d e}(z)$ by solving Eq.(31) into Eq .(34), the function $E(z)$ can be obtained. 
TABLE I: The minimum of $\chi^{2}$ by using only the Union2.1 SNIa data; for comparison, $\chi_{\Lambda \mathrm{CDM}}^{2}=562.227$.

\begin{tabular}{|c|c|c|c|c|c|c|c|c|}
\hline & $\mathrm{n}=0$ & $\mathrm{n}=1$ & $\mathrm{n}=2$ & $\mathrm{n}=3$ & $\mathrm{n}=4$ & $\mathrm{n}=5$ & $\mathrm{n}=6$ & $\mathrm{n}=7$ \\
\hline $\mathrm{m}=\mathrm{n}-1$ & 562.227 & 562.242 & 562.657 & 563.212 & 563.751 & 564.244 & 564.686 & 565.083 \\
\hline $\mathrm{m}=\mathrm{n}-2$ & - & - & 568.794 & 570.957 & 572.680 & 574.088 & 575.264 & 576.265 \\
\hline $\mathrm{m}=\mathrm{n}-3$ & - & - & - & 583.096 & 585.780 & 587.928 & 589.696 & 591.183 \\
\hline $\mathrm{m}=\mathrm{n}-4$ & - & - & - & - & 600.560 & 603.214 & 605.394 & 607.223 \\
\hline
\end{tabular}

TABLE II: The best-fit results of some models with $n-m=1$ by using only the Union2.1 SNIa data

\begin{tabular}{|c|c|c|c|c|c|c|c|c|}
\hline & $\mathrm{n}=0$ & $\mathrm{n}=1$ & $\mathrm{n}=2$ & $\mathrm{n}=3$ & $\mathrm{n}=4$ & $\mathrm{n}=5$ & $\mathrm{n}=6$ & $\mathrm{n}=7$ \\
\hline$\chi^{2}$ & 562.227 & 562.242 & 562.657 & 563.212 & 563.751 & 564.244 & 564.686 & 565.083 \\
\hline$d$ & $\gtrsim O(10)$ & 0.874 & 0.460 & 0.307 & 0.229 & 0.181 & 0.150 & 0.127 \\
\hline$\Omega_{m 0}$ & 0.278 & 0.246 & 0.272 & 0.287 & 0.298 & 0.305 & 0.311 & 0.316 \\
\hline$w_{d e 0}$ & $\sim-1.000$ & -0.997 & -1.098 & -1.167 & -1.223 & -1.266 & -1.302 & -1.332 \\
\hline
\end{tabular}

Of course, it is impossible to perform the best-fit analyses on all single-parameter models corresponding to the cases $n>m \geq 0$. However, we can still learn something from the best-fit analyses on a sample of models. Here, we are going to focus on the models with subjecting to $0 \leq n \leq 7$ as well as $\max (0, n-4) \leq m<n$ and to figure out some general results. The $\eta \mathrm{HDE}$ model corresponding to the case $(n=0, m=-1)$ is also included.

In Table $[\Pi]$, we present the best-fit $\chi^{2}$ results by using only the Union2.1 compilation of 580 supernova Ia (SNIa) data [50]. For comparison, we also fit the $\Lambda \mathrm{CDM}$ model to the same observational data, and find that the minimal $\chi_{\Lambda \mathrm{CDM}}^{2}=562.227$ for the best fit parameter $\Omega_{m 0}=0.278$. Obviously, the $\Lambda \mathrm{CDM}$ model fits to the SNIa data best. It is interesting to note that the best-fit result of the $\eta \mathrm{HDE}$ model is the same as the $\Lambda \mathrm{CDM}$ model. Actually, the $\eta \mathrm{HDE}$ model reduces to the $\Lambda$ CDM model when model parameter $d \rightarrow \infty[1]$. From Table [I], when focusing on the single-parameter models with $n>m \geq 0$, we see that models with $n-m=1$ have much smaller best-fit $\chi^{2}$ functions than models with $n-m>1$ for the given $n$. Also for the given $n-m$, the best-fit $\chi^{2}$ function increases with $n$.

It is noticed that the single-parameter models characterized by the conformal-age-like length 
TABLE III: The best-fit $\chi^{2}$ by using SNIa+BAO+CMB data sets; for comparison, $\chi_{\Lambda \mathrm{CDM}}^{2}=562.531$.

\begin{tabular}{|c|c|c|c|c|c|c|c|c|}
\hline & $\mathrm{n}=0$ & $\mathrm{n}=1$ & $\mathrm{n}=2$ & $\mathrm{n}=3$ & $\mathrm{n}=4$ & $\mathrm{n}=5$ & $\mathrm{n}=6$ & $\mathrm{n}=7$ \\
\hline $\mathrm{m}=\mathrm{n}-1$ & 562.383 & 566.363 & 562.872 & 563.738 & 565.723 & 567.922 & 570.057 & 572.039 \\
\hline $\mathrm{m}=\mathrm{n}-2$ & - & - & 636.152 & 650.851 & 661.528 & 669.697 & 676.188 & 681.495 \\
\hline $\mathrm{m}=\mathrm{n}-3$ & - & - & - & 737.659 & 749.054 & 757.655 & 764.430 & 769.934 \\
\hline $\mathrm{m}=\mathrm{n}-4$ & - & - & - & - & 813.612 & 821.699 & 828.098 & 833.314 \\
\hline
\end{tabular}

TABLE IV: The best-fit results at $1 \sigma(68.3 \%)$ and $2 \sigma(95.4 \%)$ confidence levels with SNIa+BAO+CMB data sets; for $\Lambda$ CDM model, $\chi_{\Lambda \mathrm{CDM}}^{2}=562.531$ and $\Omega_{m 0}=0.273_{-0.013}^{+0.014}{ }_{-0.026}^{0.028}$.

\begin{tabular}{|c|c|c|c|c|c|}
\hline & $(n=0, m=-1)$ & $(n=1, m=0)$ & $(n=2, m=1)$ & $(n=3, m=2)$ & $(n=4, m=3)$ \\
\hline$\chi^{2}$ & 562.383 & 566.363 & 562.872 & 563.738 & 565.723 \\
\hline$d$ & $34.5_{-27.1}^{+\infty}{ }_{-29.4}^{+\infty}$ & $0.833_{-0.018}^{+0.018}+0.037$ & $0.459_{-0.009}^{+0.009+0.017}-0.017$ & $0.310_{-0.005-0.005+0.010}^{+0.011}$ & $0.232_{-0.004}^{+0.004+0.0008}$ \\
\hline$\Omega_{m 0}$ & $0.272_{-0.021+0.022+0.036}^{+0.033}$ & $0.267_{-0.013-0.013+0.026}^{+0.024}$ & $0.275_{-0.012}^{+0.013}{ }_{-0.024}^{+0.025}$ & $0.282_{-0.012-0.012+0.025}^{+0.023}$ & $0.288_{-0.012-0.013+0.025}^{+0.023}$ \\
\hline$w_{d e 0}$ & $-0.984_{-0.016-0.060}^{+0.066}$ & $-0.981_{-0.009}^{+0.009+0.018}$ & $-1.095_{-0.013}^{+0.013+0.026}+0.025$ & $-1.176_{-0.015-0.015+0.031}^{+0.031}$ & $-1.237_{-0.018}^{+0.018}+0.035$ \\
\hline
\end{tabular}
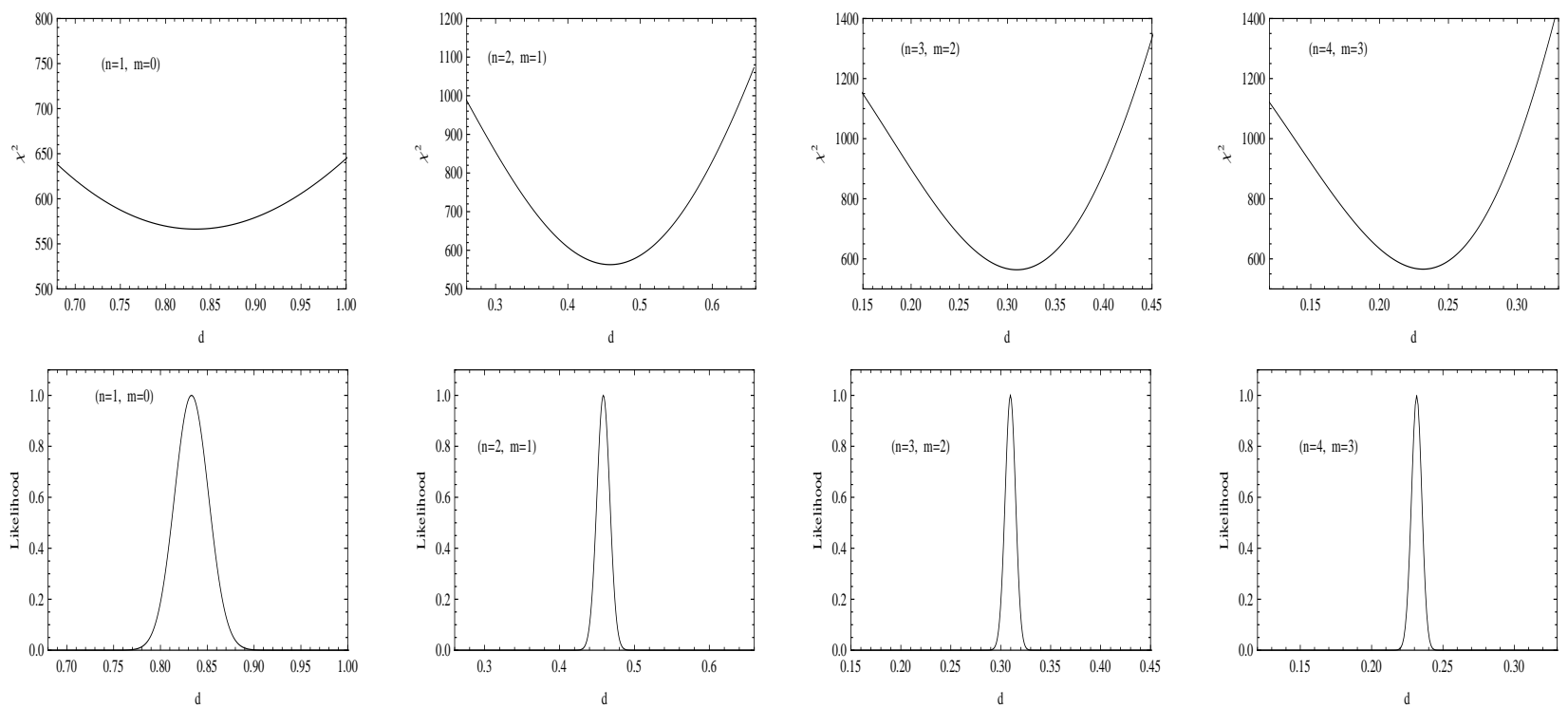

FIG. 1: The $\chi^{2}$ functions and corresponding likelihoods of models by using SNIa+BAO+CMB data sets. 

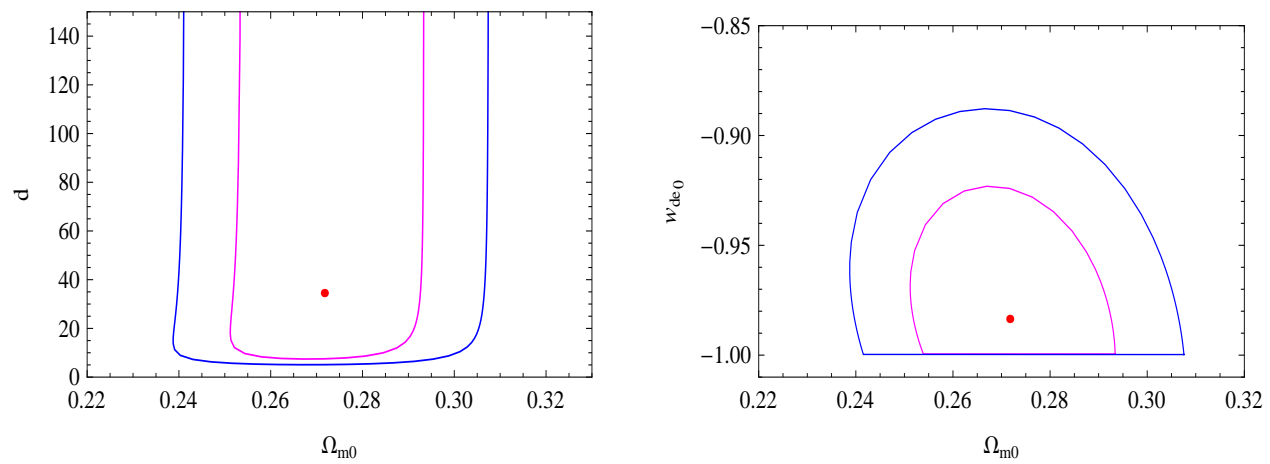

FIG. 2: Probability contours at $68.3 \%$ and $95.4 \%$ confidence levels of $\eta$ HDE model with $(n=0, m=-1)$; the best-fit values are $d=34.5$ and $\Omega_{m 0}=0.272$ by using SNIa+BAO+CMB data sets.

scale with $n-m=1$ seem to be more favored, we shall pay more attention to those models. The best-fit results for those models with some corresponding quantities are summarized in Table [I]]. We can also see that the present EoS of dark energy $w_{d e 0}$ deviating from -1 become much more for larger $n$. Among the single-parameter models $(n \geq 1)$ the present fraction of matter $\Omega_{m 0}$ is increased with larger $n$. It is interesting to note that the parameter $d$ is compatible with $1 / n$ in Table [II]. This can be enlightened by the EoS given in Eq.(10). The present EoS of dark energy is $w_{d e 0}(a=1)=-1-\frac{2}{3} n+\frac{2}{3 d} \sqrt{\Omega_{d e 0}}$. As $\Omega_{d e 0} \lesssim 1$, it leads to $d$ being compatible with $1 / n$ in order to get $w_{d e 0}$ deviating less from -1 which seems to be more favored by the observations.

The complimentary data sets from different cosmological observations often constrain dark energy models better. Therefore, we would like to perform joint analyses on the same sample of models by using the Union2.1 compilation of 580 supernova Ia (SNIa) data [50], the parameter $A$ from BAO measurements [51] and the shift parameter $R$ from CMB measurements [52]. In Table [III], we show the best-fit $\chi^{2}$ results for those models. For the given $n$, we again conclude that models with $n-m=1$ are more favored. For the given $n-m$, the best-fit $\chi^{2}$ function increases generally with $n$ except that the best-fit $\chi^{2}$ of the model with $(n=1, m=0)$ is larger than the model with $(n=2, m=1)$. This is mainly because the model with $(n=1, m=0)$ favors much smaller fraction of matter and goes more against the BAO and CMB observations which are more sensitive to the present fraction of matter than the SNIa data.

The best-fit analysis indicates that the models characterized by the conformal-age-like length $L=\frac{1}{a^{n}(t)} \int_{0}^{t} d t^{\prime} a^{n-1}\left(t^{\prime}\right)$ with small $n$ are more favored from the cosmological observations. In Table IV] we present the best-fit results of models with $n-m=1$ as well as $0 \leq n \leq 4$ at the 
$68.3 \%(95.4 \%)$ confidence level by using SNIa +BAO+CMB data sets. For the single-parameter model, $\Delta \chi^{2} \equiv \chi^{2}-\chi_{\text {min }}^{2} \leq 1.0(4.0)$ is used to constrain the model parameter at the $68.3 \%(95.4 \%)$ confidence level, while $\Delta \chi^{2} \leq 2.3(6.17)$ is used for the two-parameter model. The likelihoods or probability contours of these models are plotted in figure 1 and 2 . From Table IV, we find that the present EoS of the dark energy $w_{d e 0}$ is around -1 for model with $(n=1, m=0)$, while for models with $n>1$ the present EoS of dark energy are significantly below -1 at two $2 \sigma$ level. It is also noticed that the EoS of dark energy in the $\eta$ HDE model has $w_{d e 0} \gtrsim-1$ and slightly deviates from -1 . In Table IV, just as in Table II, one notices again that the model parameter $d$ is around $d \sim O(1 / n)$ in those four single-parameter models. It seems to hold for models with $n=m+1>0$. By referring to Eq.(18), the holographic type dark energy in such single-parameter models characterized by the conformal-age-like length $L=\frac{1}{a^{n}(t)} \int_{0}^{t} d t^{\prime} a^{n-1}\left(t^{\prime}\right)$ with small positive $n$ can be ignored in early universe as $\Omega_{d e} \simeq \frac{d^{2}}{4}\left(2 n+3 w_{m}+1\right)^{2} a^{2}$ when $a \ll 1$, and the conformalage-like length is given by $L \propto \frac{1}{a H}$.

\section{CONCLUSION AND DISCUSSION}

We have investigated a general class of holographic type dark energy models described by the characteristic length scale $L=\frac{1}{a^{n}(t)} \int_{0}^{t} d t^{\prime} a^{m}\left(t^{\prime}\right)$ with integers $n, m$. It has been shown that the recent cosmic accelerated expansion requires $n \geq 0$. For $n \geq 0$ and $m<0$, we have the fraction of dark energy density scales as $\Omega_{d e} \propto a^{3\left(1+w_{m}\right)+2 n}$ with a tiny proportionality coefficient and $w_{d e} \simeq-1-\frac{2 n}{3}$ in the early universe when $a \ll 1$. There are in general two model parameters which can be chosen as the parameter $d$ and the present fraction of dark energy $\Omega_{d e 0}$. For $n=0$ and $m<0$, the holographic type dark energy has been found to behave like a cosmological constant and there is actually only one effective parameter $\Omega_{d e 0}$. For $n>m \geq 0$, we have $\Omega_{d e} \simeq \frac{d^{2}}{4}\left(2 m+3 w_{m}+3\right)^{2} a^{2(n-m)}$ and $w_{d e} \simeq-\frac{2}{3}(n-m)+w_{m}$ in the early universe when $a \ll 1$ except that $\Omega_{d e} \simeq d^{2} a^{2 n}\left(\ln \left(\frac{a}{a_{i}}\right)\right)^{-2}$ during inflation for $m=0$. For those cases, the fraction of dark energy can be ignored in early universe when $a \ll 1$ as long as the parameter $d$ takes a normal value. Moreover, due to the analytic feature in early universe, the models with $n>m \geq 0$ have been found to be singleparameter models like the $\Lambda$ CDM model. Particularly, the EoS of the dark energy in the models with $n=m+1>0$ have been shown to transit from $w_{d e}>-1$ in the radiation- and matterdominated epoch to $w_{d e}<-1$ eventually. It has been demonstrated that the choices $n=m \geq 0$ should be abandoned as the dark energy cannot dominate the universe forever and there might be 
too large fraction of dark energy in early universe, while the choices $m>n \geq 0$ must be forbidden from the self-consistent requirement that $\Omega_{d e} \ll 1$ when $a \ll 1$.

It is worth to point out that although the energy density of the dark energy in those selfconsistent models grows or falls more slowly than the energy density of radiation and matter, the dark energy cannot dominate the early universe due to the much smaller initial density, e.g. for the models with $n=m+1>0$, we have $\rho_{d e}\left(a_{e}\right)=\Omega_{d e}\left(a_{e}\right) 3 M_{p}^{2} H_{e}^{2} \sim \rho_{r}\left(a_{e}\right) \cdot a_{e}^{2} \ll \rho_{r}\left(a_{e}\right)$ at the end of the inflation at $a_{e} \ll 1$. But the dark energy will eventually dominate the universe and be responsible for the recent cosmic acceleration. Interestingly, for models with $n>m \geq 0$, the pre-inflation part of the characteristic length $L$ is redshifted by the inflation which results in that $\Omega_{d e}\left(a_{e}\right)$ is determined by the model parameter $d$ and the inflation approximately. This means that the coincident problem of dark energy might be solved by the inflation naturally in these models.

The model with $(n=0, m=-1)$ and the single-parameter models corresponding to cases $n>$ $m \geq 0$ have been studied by using the recent cosmological observations. It has been shown that the five models with $(n=0, m=-1),(n=1, m=0),(n=2, m=1),(n=3, m=2)$ and $(n=4, m=3)$ fit to observations well. In the case $(n=0, m=-1)$, the characteristic length scale $L$ is dominated by the primordial part generated by the inflation, which resulted in small and almost constant dark energy density [1]. While for the single-parameter models characterized by the conformalage-like length $L=\frac{1}{a^{n}(t)} \int_{0}^{t} d t^{\prime} a^{n-1}\left(t^{\prime}\right)$ with $n>0$, it can be seen that the characteristic length scale behaves as $L \propto \frac{1}{H a}$ in early universe, and the best-fit analysis leads the model parameter $d$ to be $d \sim O(1 / n)$. Thus, the holographic type dark energy in such single-parameter models characterized by the conformal-age-like length $L=\frac{1}{a^{n}(t)} \int_{0}^{t} d t^{\prime} a^{n-1}\left(t^{\prime}\right)$ with small positive $n$ can be ignored in early universe as $\Omega_{d e} \simeq \frac{d^{2}}{4}\left(2 n+3 w_{m}+1\right)^{2} a^{2}$ when $a \ll 1$. The best-fit analysis has shown that the models characterized by the conformal-age-like length $L=\frac{1}{a^{n}(t)} \int_{0}^{t} d t^{\prime} a^{n-1}\left(t^{\prime}\right)$ with small $n$ are more favored from the cosmological observations.

\section{Acknowledgements}

We would like to thank R.G. Cai, M. Li, Y. Ling, J.X. Lu for useful discussions. The author (Z.P.H) would like to thank M. Q. Huang and M. Zhong for their helpful support. This work is supported in part by the National Basic Research Program of China (973 Program) under Grants No. 2010CB833000; the National Nature Science Foundation of China (NSFC) under Grants No. 10975170, 10975184, 10947016. 


\section{Appendix A: Observational data and analysis method}

In this appendix, we present the method used for the best-fit analysis on the observational data including Type Ia Supernovae (SNIa), Baryon Acoustic Oscillations (BAO), Cosmic Microwave Background $(\mathrm{CMB})$ radiation.

For only Type Ia Supernovae (SNIa) observation, the likelihood function is given by

$$
\mathcal{L}_{\mathrm{SN}}=\exp \left[-\tilde{\chi}_{\mathrm{SN}}^{2} / 2\right]
$$

For the three independent observations, the likelihood function of a joint analysis is

$$
\begin{aligned}
\mathcal{L} & =\mathcal{L}_{\mathrm{SN}} \times \mathcal{L}_{\mathrm{BAO}} \times \mathcal{L}_{\mathrm{CMB}} \\
& =\exp \left[-\left(\tilde{\chi}_{\mathrm{SN}}^{2}+\chi_{\mathrm{BAO}}^{2}+\chi_{\mathrm{CMB}}^{2}\right) / 2\right] .
\end{aligned}
$$

The model parameters yielding a minimal $\sum_{i} \chi_{i}^{2}$ and a maximal $\mathcal{L}$ will be favored by the observations. In the following, we present the calculation for the various $\chi_{i}^{2}$ of each observational data set.

\section{Type Ia Supernovae (SN Ia)}

We consider the Supernova Cosmology Project (SCP) Union2.1 compilation [50], which compiles the distance modulus $\mu_{\mathrm{obs}}\left(z_{i}\right)$ of 580 supernovae. The theoretical distance modulus is defined as

$$
\mu_{\mathrm{th}}\left(z_{i}\right) \equiv 5 \log _{10} D_{L}\left(z_{i}\right)+\mu_{0}
$$

in which the parameter $\mu_{0}$ is a nuisance parameter but is independent of the data. The Hubble-free luminosity distance is given by

$$
D_{L}(z)=(1+z) \int_{0}^{z} \frac{d z^{\prime}}{E\left(z^{\prime}\right)},
$$

with $E(z) \equiv H(z) / H_{0}$.

The $\chi^{2}$ for the SNIa data is given by

$$
\chi_{\mathrm{SN}}^{2}=\sum_{i} \frac{\left[\mu_{\mathrm{obs}}\left(z_{i}\right)-\mu_{\mathrm{th}}\left(z_{i}\right)\right]^{2}}{\sigma_{i}^{2}},
$$


where $\mu_{\mathrm{obs}}\left(z_{i}\right)$ and $\sigma_{i}$ are the observed value and the corresponding $1 \sigma$ error of distance modulus for each supernova, respectively. To reduce the effect of nuisance parameter $\mu_{0}$ [54], one can expand $\chi_{\mathrm{SN}}^{2}$ with respect to $\mu_{0}$ as follows

$$
\chi_{\mathrm{SN}}^{2}=A-2 \mu_{0} B+\mu_{0}^{2} C,
$$

where

$$
\begin{gathered}
A=\sum_{i} \frac{\left[\mu_{\mathrm{obs}}\left(z_{i}\right)-\mu_{\mathrm{th}}\left(z_{i} ; \mu_{0}=0\right)\right]^{2}}{\sigma_{i}^{2}} \\
B=\sum_{i} \frac{\mu_{\mathrm{obs}}\left(z_{i}\right)-\mu_{\mathrm{th}}\left(z_{i} ; \mu_{0}=0\right)}{\sigma_{i}^{2}} \\
C=\sum_{i} \frac{1}{\sigma_{i}^{2}}
\end{gathered}
$$

Evidently, $\chi_{\mathrm{SN}}^{2}$ has a minimum as

$$
\tilde{\chi}_{\mathrm{SN}}^{2}=A-\frac{B^{2}}{C}
$$

which is independent of $\mu_{0}$. Since $\tilde{\chi}_{\mathrm{SN}, \min }^{2}=\chi_{\mathrm{SN}, \min }^{2}$, we will adopt $\tilde{\chi}_{\mathrm{SN}}^{2}$ in our best-fit analysis.

\section{Baryon Acoustic Oscillations (BAO)}

From the distribution of SDSS luminous red galaxies, the quantity $A$ via the measurement of the BAO peak is defined as [51]

$$
A \equiv \Omega_{m 0}^{1 / 2} E\left(z_{b}\right)^{-1 / 3}\left(\frac{1}{z_{b}} \int_{0}^{z_{b}} \frac{d z^{\prime}}{E\left(z^{\prime}\right)}\right)^{2 / 3},
$$

at the redshift $z_{b}=0.35$. The SDSS BAO measurement [51] gives $A_{\mathrm{obs}}=0.469\left(n_{s} / 0.98\right)^{-0.35} \pm$ 0.017, where the scalar spectral index is taken to be $n_{s}=0.968$ from the WMAP7 measurement [52]. The $\chi^{2}$ of the BAO data is given by:

$$
\chi_{\mathrm{BAO}}^{2}=\frac{\left(A-A_{\mathrm{obs}}\right)^{2}}{0.017^{2}} .
$$

\section{Cosmic Microwave Background (CMB)}

The shift parameter $R$ is defined by [53]

$$
R \equiv \Omega_{m 0}^{1 / 2} \int_{0}^{z_{*}} \frac{d z^{\prime}}{E\left(z^{\prime}\right)},
$$


where the redshift of the recombination $z_{*}=1091.3$ WMAP7 [52]. The shift parameter $R$, which relates the angular diameter distance to the last scattering surface, the comoving size of the sound horizon at $z_{*}$ and the angular scale of the first acoustic peak in CMB power spectrum of temperature, has been measured to be $1.725 \pm 0.018$ [52]. The $\chi^{2}$ of the CMB data is given by:

$$
\chi_{\mathrm{CMB}}^{2}=\frac{(R-1.725)^{2}}{0.018^{2}} \text {. }
$$

[1] Z. -P. Huang and Y. -L. Wu, arXiv:1202.4228 [gr-qc], to be published in PRD.

[2] A. G. Riess et al. [Supernova Search Team Collaboration], Astron. J. 116, 1009 (1998) [arXiv:astro-ph/9805201].

[3] S. Perlmutter et al. [Supernova Cosmology Project Collaboration], Astrophys. J. 517, 565 (1999) [arXiv:astro-ph/9812133].

[4] V. Sahni and A. A. Starobinsky, Int. J. Mod. Phys. D 9, 373 (2000)

[5] P. J. E. Peebles and B. Ratra, Rev. Mod. Phys. 75, 559 (2003) [astro-ph/0207347].

[6] T. Padmanabhan, Phys. Rept. 380, 235 (2003) [hep-th/0212290].

[7] E. J. Copeland, M. Sami and S. Tsujikawa, Int. J. Mod. Phys. D 15, 1753 (2006) [arXiv:hep-th/0603057].

[8] S. Tsujikawa, arXiv:1004.1493 [astro-ph.CO].

[9] For a review, see: M. Li, X. -D. Li, S. Wang, Y. Wang, Commun. Theor. Phys. 56, 525-604 (2011) [arXiv:1103.5870 [astro-ph.CO]], and references therein.

[10] S. Weinberg, Rev. Mod. Phys. 61, 1-23 (1989); arXiv:astro-ph/0005265].

[11] G. 't Hooft, arXiv:gr-qc/9310026.

[12] L. Susskind, J. Math. Phys. 36, 6377-6396 (1995) [arXiv:hep-th/9409089].

[13] A. G. Cohen, D. B. Kaplan, A. E. Nelson, Phys. Rev. Lett. 82, 4971-4974 (1999) [arXiv:hep-th/9803132].

[14] P. Horava and D. Minic, Phys. Rev. Lett. 85, 1610 (2000) [hep-th/0001145].

[15] S. D. Thomas, Phys. Rev. Lett. 89, 081301 (2002).

[16] W. Fischler and L. Susskind, hep-th/9806039

[17] R. Bousso, JHEP 9907, 004 (1999) [hep-th/9905177].

[18] A. H. Guth, Phys. Rev. D 23, 347-356 (1981). 
[19] A. A. Starobinsky, Phys. Lett. B 91, 99 (1980)

[20] A. D. Linde, Phys. Lett. B 108, 389-393 (1982).

[21] A. Albrecht, P. J. Steinhardt, Phys. Rev. Lett. 48, 1220-1223 (1982).

[22] Z. -P. Huang and Y. -L. Wu, arXiv:1202.2590 [hep-th], to be published.

[23] Z. -P. Huang and Y. -L. Wu, arXiv:1202.3517][astro-ph.CO].

[24] S. D. H. Hsu, Phys. Lett. B 594, 13 (2004) [hep-th/0403052].

[25] M. Li, Phys. Lett. B 603, 1 (2004) [arXiv:hep-th/0403127].

[26] Q. -G. Huang and M. Li, JCAP 0408, 013 (2004) [astro-ph/0404229].

[27] Y. -g. Gong, Phys. Rev. D 70, 064029 (2004) [hep-th/0404030].

[28] Y. S. Myung, Phys. Lett. B 610, 18 (2005) [hep-th/0412224].

[29] D. Pavon and W. Zimdahl, Phys. Lett. B 628, 206 (2005) [gr-qc/0505020].

[30] B. Wang, Y. -g. Gong and E. Abdalla, Phys. Lett. B 624, 141 (2005) [hep-th/0506069].

[31] S. 'I. Nojiri and S. D. Odintsov, Gen. Rel. Grav. 38, 1285 (2006) [hep-th/0506212].

[32] B. Hu and Y. Ling, Phys. Rev. D 73, 123510 (2006) [hep-th/0601093].

[33] B. Chen, M. Li and Y. Wang, Nucl. Phys. B 774, 256 (2007) astro-ph/0611623].

[34] H. Li, Z. -K. Guo and Y. -Z. Zhang, Int. J. Mod. Phys. D 15, 869 (2006) [astro-ph/0602521].

[35] M. Setare, Phys. Lett. B 642, 1 (2006) [hep-th/0609069].

[36] R. -G. Cai, Phys. Lett. B 657, 228-231 (2007) [arXiv:0707.4049 [hep-th]].

[37] H. Wei, R. -G. Cai, Phys. Lett. B 660, 113-117 (2008) [arXiv:0708.0884][astro-ph]].

[38] C. Gao, X. Chen, Y. -G. Shen, Phys. Rev. D 79, 043511 (2009) [arXiv:0712.1394 [astro-ph]].

[39] L. N. Granda and A. Oliveros, Phys. Lett. B 669, 275 (2008) [arXiv:0810.3149 [gr-qc]].

[40] L. N. Granda and A. Oliveros, Phys. Lett. B 671, 199 (2009) [arXiv:0810.3663 [gr-qc]].

[41] Y. Gong and T. Li, Phys. Lett. B 683, 241 (2010) [arXiv:0907.0860 [hep-th]].

[42] M. Jamil, E. N. Saridakis and M. R. Setare, Phys. Lett. B 679, 172 (2009) [arXiv:0906.2847 [hep-th]].

[43] C. -J. Feng and X. Zhang, Phys. Lett. B 680, 399 (2009) [arXiv:0904.0045 [gr-qc]].

[44] S. del Campo, J. .C. Fabris, R. Herrera and W. Zimdahl, Phys. Rev. D 83, 123006 (2011) [arXiv:1103.3441] [astro-ph.CO]].

[45] C. Gao, arXiv:1108.5827 [gr-qc].

[46] Z. Zhang, M. Li, X. -D. Li, S. Wang and W. -S. Zhang, arXiv:1202.5163 [astro-ph.CO].

[47] H. Wei, arXiv:1204.4032 [gr-qc].

[48] Y. Ling and W. J. Pan, arXiv:1205.0209 [gr-qc]. 
[49] K. A. Olive, G. Steigman and T. P. Walker, Phys. Rept. 333, 389 (2000) [arXiv:astro-ph/9905320].

[50] N. Suzuki, D. Rubin, C. Lidman, G. Aldering, R. Amanullah, K. Barbary, L. F. Barrientos and J. Botyanszki et al., Astrophys. J. 746, 85 (2012) [arXiv:1105.3470 [astro-ph.CO]].

[51] D. J. Eisenstein et al. [SDSS Collaboration], Astrophys. J. 633, 560 (2005) [arXiv:astro-ph/0501171].

[52] E. Komatsu et al. [WMAP Collaboration], Astrophys. J. Suppl. 192, 18 (2011) [arXiv:1001.4538 [astro-ph.CO]].

[53] J. R. Bond, G. Efstathiou and M. Tegmark, Mon. Not. Roy. Astron. Soc. 291, L33 (1997) [arXiv:astro-ph/9702100].

[54] L. Perivolaropoulos, Phys. Rev. D 71, 063503 (2005) [arXiv:astro-ph/0412308]. 